\title{
Treatment decision for cancer patients with fever during the coronavirus disease 2019 (COVID-19) pandemic
}

\author{
In Hee Lee ${ }^{1}$, Sung Ae $\mathrm{Koh}^{2}$, Soo Jung Lee ${ }^{1}$, Sun Ah Lee ${ }^{3}$, Yoon Young Cho ${ }^{4}$, Ji Yeon Lee ${ }^{5}$, Jin Young Kim ${ }^{6}$ \\ ${ }^{1}$ Department of Oncology/Hematology, School of Medicine, Kyungpook National University, Kyungpook National University Chilgok Hospital, \\ Daegu, Korea \\ ${ }^{2}$ Department of Hematology-Oncology, Yeungnam University College of Medicine, Daegu, Korea \\ ${ }^{3}$ Department of Oncology/Hematology, Daegu Fatima Hospital, Daegu, Korea \\ ${ }^{4}$ Division of Hematology/Oncology, Department of Internal Medicine, Daegu Catholic University Shcool of Medicine, Daegu, Korea \\ ${ }^{5}$ Department of Infectious Diseases, Keimyung University Dongsan Medical Center, Daegu, Korea \\ ${ }^{6}$ Division of Hematology/Oncology, Department of Internal Medicine, Keimyung University Dongsan Medical Center, Daegu, Korea
}

Received: May 18, 2021

Revised: July 26, 2021

Accepted: July 29, 2021

Corresponding author:

Jin Young Kim, MD, PhD

Division of Hematology/Oncology,

Department of Internal Medicine,

Keimyung University Dongsan

Medical Center, 1035 Dalgubeol-

daero, Dalseo-gu, Daegu 42601,

Korea

Tel: +82-53-258-6670

Fax: +82-53-425-6476

E-mail: takgu@dsmc.or.kr
Background: Cancer patients have been disproportionally affected by the coronavirus disease 2019 (COVID-19) pandemic, with high rates of severe outcomes and mortality. Fever is the most common symptom in COVID-19 patients. During the COVID-19 pandemic, physicians may have difficulty in determining the cause of fever (COVID-19, another infection, or cancer fever) in cancer patients. Furthermore, there are no specific guidelines for managing cancer patients with fever during the COVID-19 pandemic. Thus, this study evaluated the clinical characteristics and outcomes of cancer patients with fever during the COVID-19 pandemic.

Methods: This study retrospectively reviewed the medical records of 328 cancer patients with COVID-19 symptoms (fever) admitted to five hospitals in Daegu, Korea from January to October 2020. We obtained data on demographics, clinical manifestations, laboratory test results, chest computed tomography images, cancer history, cancer treatment, and outcomes of all enrolled patients from electronic medical records.

Results: The most common COVID-19-like symptoms were fever $(n=256,78 \%)$. Among 256 patients with fever, only three (1.2\%) were diagnosed with COVID-19. Most patients $(253,98.8 \%)$ with fever were not diagnosed with COVID-19. The most common solid malignancies were lung cancer $(65,19.8 \%)$ and hepatobiliary cancer $(61,18.6 \%)$. Twenty patients with fever experienced a delay in receiving cancer treatment. Eighteen patients discontinued active cancer treatment because of fever. Major events during the treatment delay period included death (2.7\%), cancer progression (1.5\%), and major organ dysfunction (2.7\%).

Conclusion: Considering that only $0.9 \%$ of patients tested for COVID-19 were positive, screening for COVID-19 in cancer patients with fever should be based on the physician's clinical decision, and patients might not be routinely tested.

Keywords: COVID-19; Fever; Neoplasms; Neoplastic fever 


\section{Introduction}

Since its emergence in Wuhan, China in December 2019, coronavirus disease 2019 (COVID-19) has become a pandemic [1]. Patients with cancer are susceptible to infection during the pandemic due to the effect of anticancer treatment and the immunosuppressive properties of cancer itself [2]. A Chinese study reported that approximately $1 \%$ of patients with COVID-19 had cancer, which was five-fold higher than the general cancer incidence in China [3]. Onder et al. [4] reported that approximately $20 \%$ of people who died of COVID-19 had a history of active cancer in the past 5 years. As a group at high risk of infection during the COVID-19 pandemic, physicians should pay more attention to cancer patients.

The clinical symptoms of COVID-19 include fever, cough, dyspnea, fatigue, headache, sore throat, myalgia, and diarrhea $[5,6]$. Fever is the most common symptom of COVID-19, and many patients are tested for COVID-19 due to fever [7-9]. In cancer patients, fever might be the only sign of infection, which can be life-threatening in those undergoing chemotherapy. There are other causes of fever in cancer patients besides infection. Neoplastic fever, a fever caused by cancer itself, has been reported as the most common cause of fever of unknown origin in cancer patients [10]. Since the emergence of the COVID-19 pandemic, many cancer patients have visited outpatient clinics or emergency units because of fever, and most of them have been tested for COVID-19 regardless of the possible causes of fever, including other infections and neoplastic fever. In addition, physicians sometimes face difficulty in determining the cause of the fever (COVID-19 vs. others) in cancer patients with COVID-19-like symptoms. Thus, we analyzed the clinical characteristics and outcomes of 328 patients with cancer who had fever and/or other COVID-19-like symptoms during the COVID-19 pandemic at five hospitals in Daegu.

\section{Materials and methods}

\section{Patient selection}

This study retrospectively reviewed the medical records of 328 cancer patients with COVID-19-like symptoms who had been tested for COVID-19 at five hospitals (Kyungpook National University Chilgok Hospital, Yeungnam University Medical Center, Daegu Fatima Hospital, Daegu Catholic University Medical Center, and Keimyung University Dongsan Medical Center) in Daegu from January to October 2020. COVID-19-like symptoms included fever, cough, sore throat, rhinorrhea, myalgia, and arthritis. Fever was defined as a tympanic temperature of at least $38^{\circ} \mathrm{C}$ $\left(100.4^{\circ} \mathrm{F}\right)$ [11]. All patients were tested for COVID-19. COVID-19 was diagnosed based on the criteria published by the World Health
Organization and confirmed by real-time polymerase chain reaction (PCR) assays of nasal and/or pharyngeal specimens [12-14]. COVID-19 death was defined as death resulting from a clinically compatible illness in a patient with confirmed COVID-19 unless a clear alternative cause of death that cannot be associated with COVID-19 could be proven.

\section{Data collection}

We obtained data on demographics, clinical manifestations, laboratory test results, chest computed tomography images, cancer history, cancer treatment, and outcomes of all enrolled patients from electronic medical records. Patient data were collected retrospectively using uniform database templates to ensure consistent data collection.

\section{Statistical analysis}

Statistical analysis was performed using IBM SPSS version 20.0 (IBM Corp, Armonk, NY, USA). Continuous variables are expressed as the mean \pm standard deviation or median (interquartile range), as appropriate. Categorical variables are summarized as counts and percentages in each category. Descriptive statistics were used to characterize the cohort.

\section{Results}

\section{Baseline characteristics}

From January to October 2020, 328 cancer patients with COVID19-like symptoms were admitted to five hospitals in Daegu. Table 1 shows the baseline characteristics of the patients. The median age was 67 years (range, 14-99 years) with 126 patients (38.4\%) aged $>70$ years. A total of 191 patients $(58.2 \%)$ were males. The four most common COVID-19-like symptoms were fever $(\mathrm{n}=256$, $78.0 \%)$, cough $(n=52,15.9 \%)$, diarrhea $(n=19,5.8 \%)$, and sore throat $(\mathrm{n}=6,1.8 \%)$. Comorbidities included hypertension $(\mathrm{n}=134,40.9 \%)$, diabetes mellitus $(\mathrm{n}=91,27.7 \%)$, and heart disease $(n=20,6.1 \%)$.

\section{Tumor characteristics}

The most common solid malignancies were lung ( $\mathrm{n}=65,19.8 \%)$ and hepatobiliary $(\mathrm{n}=61,18.6 \%)$ cancers (Table 2). Sixty-two patients (18.9\%) had hematologic malignancies. Most patients (91.5\%) received at least one antitumor therapy; 244 (74.4\%) received cytotoxic chemotherapy, and 111 (33.8\%) underwent surgical resection for tumor control. Based on the last response evaluation of the patients, 22 patients $(6.7 \%)$ showed complete response, 39 (11.9\%) showed partial response, 58 (17.7\%) had stable disease, and 92 (28.0\%) experienced progressive disease. 
Table 1. Characteristics of the study population

\begin{tabular}{|c|c|}
\hline Factor & Data \\
\hline Patient & $328(100)$ \\
\hline \multicolumn{2}{|l|}{ Age (yr) } \\
\hline$\leq 70$ & 202 (61.6) \\
\hline$>70$ & $126(38.4)$ \\
\hline \multicolumn{2}{|l|}{ Sex } \\
\hline Male & $191(58.2)$ \\
\hline Female & $137(41.8)$ \\
\hline \multicolumn{2}{|l|}{ ECOG PS } \\
\hline 0 & $12(3.7)$ \\
\hline 1 & $146(44.5)$ \\
\hline 2 & $83(25.3)$ \\
\hline 3 & $59(18.0)$ \\
\hline 4 & $28(8.5)$ \\
\hline \multicolumn{2}{|l|}{ Comorbidity } \\
\hline Hypertension & $134(40.9)$ \\
\hline Diabetes mellitus & $91(27.7)$ \\
\hline Tuberculosis & $5(1.5)$ \\
\hline Hepatitis & $1(0.3)$ \\
\hline Hyperlipidemia & $9(2.7)$ \\
\hline COPD & $11(3.4)$ \\
\hline Heart disease & $20(6.1)$ \\
\hline Cerebrovascular disease & $11(3.4)$ \\
\hline Chronic kidney disease & $9(2.7)$ \\
\hline Chronic liver disease & $13(4.0)$ \\
\hline Organ transplantation & $9(2.7)$ \\
\hline \multicolumn{2}{|l|}{ Symptom } \\
\hline Fever & 256 (78.0) \\
\hline Cough & $52(15.9)$ \\
\hline Sore throat & $6(1.8)$ \\
\hline Rhinorrhea & $5(1.5)$ \\
\hline Diarrhea & $19(5.8)$ \\
\hline Myalgia & $17(5.2)$ \\
\hline Arthritis & $2(0.6)$ \\
\hline
\end{tabular}

Values are presented as number (\%).

ECOG PS, Eastern Cooperative Oncology Group performance status; COPD, chronic obstructive pulmonary disease.

\section{Cause of fever and treatment delay affected by fever}

Among the patients tested for COVID-19, only three (1.2\%) were confirmed to have COVID-19 based on a positive severe acute respiratory syndrome coronavirus (SARS-CoV)-2 PCR test result (Table 3). Most patients $(253,98.8 \%)$ with fever were not diagnosed with COVID-19. Of the sputum, blood, urine, and body fluid samples, $3.9 \%, 1.2 \%, 0.4 \%$, and $0.8 \%$, respectively, were culture-positive. More than $90 \%$ of the patients were neither diagnosed with COVID-19 nor culture-positive. Thirty-three patients (12.9\%) diagnosed with neutropenic fever.

Twenty patients with fever experienced a delay in receiving can-
Table 2. Tumor characteristics and cancer treatment

\begin{tabular}{|c|c|}
\hline Factor & Data $(n=328)$ \\
\hline \multicolumn{2}{|l|}{ Type of malignancy } \\
\hline \multicolumn{2}{|l|}{ Solid tumors } \\
\hline Lung cancer & 65 (19.8) \\
\hline Hepatobiliary & $61(18.6)$ \\
\hline Stomach & $16(4.9)$ \\
\hline Colorectal & $26(7.9)$ \\
\hline Breast & $19(5.8)$ \\
\hline Head and neck & $18(5.5)$ \\
\hline Gynecological & $26(7.9)$ \\
\hline Genitourinary & $19(5.8)$ \\
\hline Others ${ }^{\mathrm{a})}$ & $16(4.9)$ \\
\hline Hematological malignancies & $62(18.9)$ \\
\hline \multicolumn{2}{|l|}{ History of cancer treatment } \\
\hline Yes & $300(91.5)$ \\
\hline No & $28(8.5)$ \\
\hline \multicolumn{2}{|l|}{ Type of cancer treatment } \\
\hline Surgery & $111(33.8)$ \\
\hline Cytotoxic chemotherapy & $244(74.4)$ \\
\hline Target therapy & $61(18.6)$ \\
\hline Immune checkpoint inhibitor & $37(11.3)$ \\
\hline Radiation & $77(23.5)$ \\
\hline Others ${ }^{b)}$ & $9(2.7)$ \\
\hline \multicolumn{2}{|l|}{ Response evaluation } \\
\hline $\mathrm{CR}$ & $22(6.7)$ \\
\hline PR & 39 (11.9) \\
\hline SD & $58(17.7)$ \\
\hline PD & $92(28.0)$ \\
\hline iRECIST CR & $0(0)$ \\
\hline iRECIST PR & $0(0)$ \\
\hline iRECIST SD & $1(0.3)$ \\
\hline iRECIST PD & $1(0.3)$ \\
\hline Unknown & $115(35.1)$ \\
\hline
\end{tabular}

Values are presented as number (\%).

$C R$, complete response; PR, partial response; $S D$, stable disease; $P D$, progressive disease; iRECIST, Response Evaluation Criteria in Solid Tumors (RECIST version 1.1) in cancer immunotherapy trials.

a)Sarcoma, primary unknown cancer. ${ }^{b}$ Transarterial chemoembolization.

cer treatment, with a median delay of 17.5 days (range, 3-33 days). Eighteen patients discontinued active cancer treatment because of fever. Major events during the treatment delay period included death $(2.7 \%)$, cancer progression (1.5\%), and major organ dysfunction (2.7\%). Two patients died of COVID-19. Twenty-eight patients (8.5\%) died of cancer progression.

\section{Characteristics of cancer patients diagnosed with COVID-19}

Table 4 shows the characteristics of the patients with cancer diag- 
Table 3. Cause of fever and treatment delay affected by fever

\begin{tabular}{lc}
\hline Factor & Data $(\mathrm{n}=328)$ \\
\hline Cause of fever $\left.(\mathrm{n}=256)^{\mathrm{a}}\right)^{-}$ & \\
COVID-19 & \\
Other infection & $10(3.2)$ \\
$\quad$ Pneumonia & $3(1.2)$ \\
$\quad$ Bloodstream infection & $1(0.4)$ \\
$\quad$ Urinary tract infection & $2(0.8)$ \\
$\quad$ Body fluid infection ${ }^{b)}$ & $33(12.9)$ \\
Neutropenic fever & \\
Delay of cancer treatment due to fever & $20(6.1)$ \\
Delay & $18(5.5)$ \\
Discontinuation & $290(88.4)$ \\
No & \\
Major event during delay period & $9(2.7)$ \\
Death & $5(1.5)$ \\
Cancer progression & $17(5.2)$ \\
Delay of admission & $9(2.7)$ \\
Major organ dysfunction & \\
Cause of death & $2(0.6)$ \\
COVID-19 & $28(8.5)$ \\
Cancer progression & $3(0.9)$ \\
Sepsis & $5(1.5)$ \\
Pneumonia & $3(0.9)$ \\
\hline Hemorrhage &
\end{tabular}

Values are presented as number (\%).

COVID-19, coronavirus disease 2019.

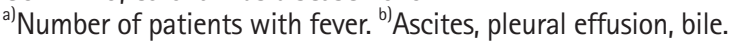

nosed with COVID-19. Of the three patients, two died. Two patients were identified as members of a religious group called 'Shincheonji.' A 71-year-old man with hypertension, chronic obstructive pulmonary disease, and heart disease was diagnosed with stage II lung cancer in January 2020. He presented with COVID-19 pneumonia after undergoing cytotoxic chemotherapy and died of COVID-19. An 82-year-old man with a history of laryngeal cancer was diagnosed with COVID-19 and recovered. A 73-year-old woman was simultaneously diagnosed with COVID-19 and lymphoma and died of COVID-19.

\section{Discussion}

Cancer patients are a vulnerable population during the ongoing COVID-19 pandemic [15-17]. They are at a high risk of SARSCoV-2 infection and are more likely than other COVID-19 patients to develop severe disease [2]. In this study, we analyzed 328 cancer patients admitted to five hospitals in Daegu to investigate the incidence of COVID-19 in cancer patients with COVID-19like symptoms.

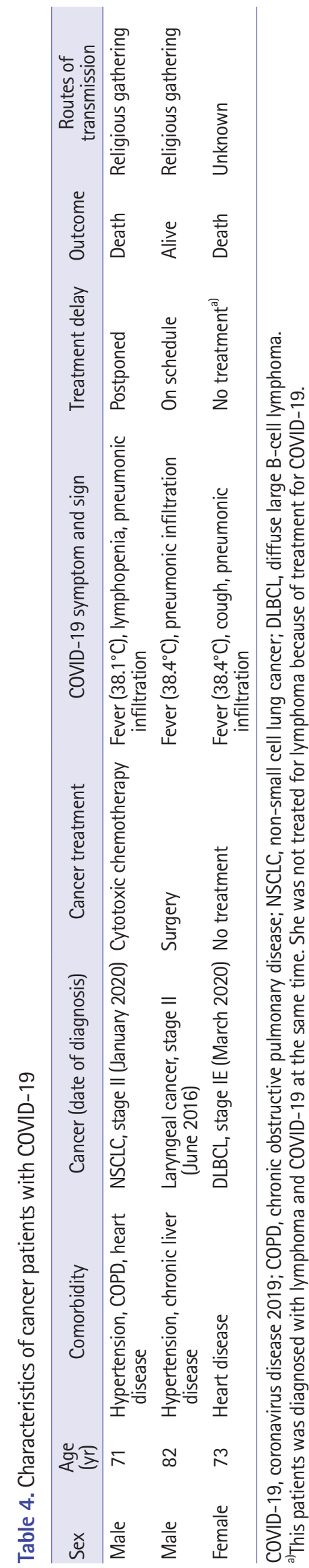


Malignancy is well known to cause fever and neoplastic fever, which are frequently encountered in febrile cancer patients without other causes of fever and presents a diagnostic challenge in differentiating whether fever is attributable to infection, therapy, or disease [10]. In our study, the most common COVID-19-like symptom was fever. However, among the 328 patients with COVID-related symptoms, only three patients were diagnosed with COVID-19 (0.9\%). The cause of fever in patients without COVID-19 or positive culture results was considered to be neoplastic fever. It may be challenging to differentiate COVID-19 from other infections and neoplastic fever. However, some factors that may be helpful in discerning between COVID-19 infection and other causes of fever are as follows: (1) COVID-19-related fever usually occurs between 2 and 14 days following exposure. (2) Neoplastic fever frequently occurs in patients with specific cancer subtypes such as lymphoma, leukemia, renal cell carcinoma, and sarcoma. (3) The most common symptoms that occur with neoplastic fever are diaphoresis, but less often include chills and rigor. In contrast, infectious fevers tend to present with warmth, diaphoresis, and chills, reflective of peripheral vasodilation. (4) Hypotension and tachycardia are commonly accompanied by systemic infections caused by gram-negative organisms [18].

Approximately $15 \%$ of cancer patients with fever experienced a delay in receiving chemotherapy. Eight patients died during the treatment delay period, six patients showed cancer progression, and eight patients experienced major organ dysfunction. A total of 33 patients died during the study period. Two of them died of COVID-19, and the other died of cancer-related complications. Cancer patients receiving adjuvant or palliative chemotherapy were advised to continue their treatment following the guidelines for cancer care during the COVID-19 pandemic, and clinicians were advised to implement treatment strategies with fewer hospital visits. However, patients had a tendency to frequently visit the hospital not only because of their symptoms but also due to anxiety. Patients' anxiety and physicians' difficulty in determining the cause of fever led to excessive testing for COVID-19 [19].

Considering that only $0.9 \%$ of patients tested for COVID- 19 were positive, and the postponement of the chemotherapy schedule could lead to significant adverse events such as death or cancer progression, this study shows that an adequate screening test for COVID-19 in cancer patients with fever based on physicians' clinical decisions can improve their outcome.

The current study had certain limitations, including its retrospective nature and small sample size. However, the present results suggest that during the COVID-19 pandemic period, the approach to cancer patients with fever should be different from that before the pandemic period. Excessive testing for COVID-19 is not the best way to improve the prognosis of patients. Adequate testing for COVID-19 and adherence to COVID-19 prevention guidelines, including handwashing and wearing masks, are crucial for improving the prognosis of cancer patients during the COVID-19 pandemic.

\section{Notes}

\section{Ethical statements}

All procedures were performed in accordance with the ethical standards of the institutional and/or national research committee and with the 1964 Declaration of Helsinki and its later amendments or comparable ethical standards. The study was approved by the Institutional Review Board of five hospitals (Kyungpook National University Chilgok Hospital, Yeungnam University Medical Center, Daegu Fatima Hospital, Daegu Catholic University Medical Center, and Keimyung University Dongsan Medical Center) in Daegu (No. KNUH 2020-03-044, CR21-024, 2020-09-100, 202010-006), which waived the requirement for informed consent.

\section{Conflicts of interest}

No potential conflict of interest relevant to this article was reported.

\section{Funding}

This work was supported by the Research Program of Medicity Daegu Council funded by Daegu Metropolitan City (fund code COVID19_DM11).

\section{Author contributions}

Conceptualization: IHL, JYK; Data curation: IHL, SAK, SJL, SAL, YYC, JYL; Formal analysis: IHL, SAK, SJL, YYC, JYL, JYK; Supervision, Funding acquisition: JYK; Writing-original draft: IHL; Writing-review \& editing: SAL.

\section{ORCID}

In Hee Lee, https://orcid.org/0000-00002-0139-9768

Sung Ae Koh, https://orcid.org/0000-0001-5150-9702

Soo Jung Lee, https://orcid.org/0000-0003-0066-4109

Sun Ah Lee, https://orcid.org/0000-0002-4577-0514

Yoon Young Cho, https://orcid.org/0000-0003-0282-9683

Ji Yeon Lee, https://orcid.org/0000-0002-2788-1392

Jin Young Kim, https://orcid.org/0000-0002-9597-9834

\section{References}

\section{Zhang L, Zhu F, Xie L, Wang C, Wang J, Chen R, et al. Clinical}


characteristics of COVID-19-infected cancer patients: a retrospective case study in three hospitals within Wuhan, China. Ann Oncol 2020;31:894-901.

2. Kuderer NM, Choueiri TK, Shah DP, Shyr Y, Rubinstein SM, Rivera DR, et al. Clinical impact of COVID-19 on patients with cancer (CCC19): a cohort study. Lancet 2020;395:1907-18.

3. Liang W, Guan W, Chen R, Wang W, Li J, Xu K, et al. Cancer patients in SARS-CoV-2 infection: a nationwide analysis in China. Lancet Oncol 2020;21:335-7.

4. Onder G, Rezza G, Brusaferro S. Case-fatality rate and characteristics of patients dying in relation to COVID-19 in Italy. JAMA 2020;323:1775-6.

5. Zhao D, Yao F, Wang L, Zheng L, Gao Y, Ye J, et al. A comparative study on the clinical features of coronavirus 2019 (COVID-19) pneumonia with other pneumonias. Clin Infect Dis 2020;71:756-61.

6. Zhu J, Ji P, Pang J, Zhong Z, Li H, He C, et al. Clinical characteristics of 3062 COVID-19 patients: a meta-analysis. J Med Virol 2020;92:1902-14.

7. Wiersinga WJ, Rhodes A, Cheng AC, Peacock SJ, Prescott HC. Pathophysiology, transmission, diagnosis, and treatment of coronavirus disease 2019 (COVID-19): a review. JAMA 2020; 324:782-93.

8. Yang J, Zheng Y, Gou X, Pu K, Chen Z, Guo Q, et al. Prevalence of comorbidities and its effects in patients infected with SARSCoV-2: a systematic review and meta-analysis. Int $J$ Infect Dis 2020;94:91-5.

9. Rodriguez-Morales AJ, Cardona-Ospina JA, Gutiérrez-Ocampo E, Villamizar-Peña R, Holguin-Rivera Y, Escalera-Antezana JP, et al. Clinical, laboratory and imaging features of COVID-19: a systematic review and meta-analysis. Travel Med Infect Dis
2020;34:101623.

10. Foggo V, Cavenagh J. Malignant causes of fever of unknown origin. Clin Med (Lond) 2015;15:292-4.

11. Schneider A, Kirsten H, Lordick F, Lordick F, Lübbert C, von Braun A. Covid-19 in outpatients: is fever a useful indicator for SARS-CoV-2 infection? PLoS One 2021;16:e0246312.

12. Islam KU, Iqbal J. An update on molecular diagnostics for COVID-19. Front Cell Infect Microbiol 2020;10:560616.

13. Seyed Hosseini E, Riahi Kashani N, Nikzad H, Azadbakht J, Hassani Bafrani H, Haddad Kashani H. The novel coronavirus Disease-2019 (COVID-19): mechanism of action, detection and recent therapeutic strategies. Virology 2020;551:1-9.

14. Yüce M, Filiztekin E, Özkaya KG. COVID-19 diagnosis: a review of current methods. Biosens Bioelectron 2021;172:112 752.

15. Boutros M, Moujaess E, Kourie HR. Cancer management during the COVID-19 pandemic: choosing between the devil and the deep blue sea. Crit Rev Oncol Hematol 2021 Mar 15 [Epub]. https://doi.org/10.1016/j.critrevonc.2021.103273.

16. Hanna TP, Evans GA, Booth CM. Cancer, COVID-19 and the precautionary principle: prioritizing treatment during a global pandemic. Nat Rev Clin Oncol 2020;17:268-70.

17. Jee J, Foote MB, Lumish M, Stonestrom AJ, Wills B, Narendra V, et al. Chemotherapy and COVID-19 outcomes in patients with cancer. J Clin Oncol 2020;38:3538-46.

18. Pasikhova Y, Ludlow S, Baluch A. Fever in patients with cancer. Cancer Control 2017;24:193-7.

19. Lee JB, Jung M, Kim JH, Kim BH, Kim Y, Kim YS, et al. Guidelines for cancer care during the COVID-19 pandemic in South Korea. Cancer Res Treat 2021;53:323-9. 